Proceeding Series of the Brazilian Society of Computational and Applied Mathematics

\title{
Aproximação de Dados Através do Processo de Mínimos Quadrados
}

\author{
Ítalo F. Nunes da Paz ${ }^{1}$ \\ Centro de Tecnologia, Departamento de Engenharia Mecânica, UFRN, Natal, RN \\ Fabiana T. Santana ${ }^{2}$ \\ Escola de Ciências e Tecnologia, UFRN, Natal, RN
}

Resumo. Neste trabalho foi feito um estudo detalhado de aproximações por Mínimos Quadrados no espaço coluna de uma matriz $A$. Em particular, foi feito o desenvolvimento matemático para se obter a melhor função que se ajusta aos pontos $\left(x_{i}, y_{i}\right)$, obtidos experimentalmente, utilizando o produto interno euclidiano em $R^{m}$. Tal função é denominada aproximação de mínimos quadrados. Por fim, foi aplicado os conceitos estudados na obtenção da estimativa da população brasileira para o ano de 2020.

Palavras-chave. Mínimos Quadrados, Ajuste de Curvas, Produto Interno.

\section{Introdução}

Muitas vezes, os sistemas lineares oriundos de um modelo matemático podem não apresentar solução devido às aproximações feitas durante a modelagem do problema. Nessas situações, a Álgebra Linear fornece a melhor solução para o problema através dos mínimos quadrados, $[2,3]$. Neste trabalho, dado um conjunto de pontos $\left(x_{1}, y_{1}\right),\left(x_{2}, y_{2}\right), \ldots,\left(x_{n}, y_{n}\right)$ a curva que mais se aproxima é obtida minimizando o erro de aproximação. Isto é, a quantidade $\|\vec{e}\|=\|A \vec{x}-\vec{b}\|$ pode ser vista como uma medida de quão próximo o vetor solução $\vec{x}$ está da solução do sistema. A regressão por mínimos quadrados tem importância especial e aparece em várias aplicações físicas obtidas experimentalmete onde se possuem um conjunto de pontos, como por exemplo em determinação de constante de molas, determinação de velocidade, dentre outros [2].

\section{Obtenção de Soluções por Mínimos Quadrados e Aplicação}

No caso de mínimos quadrados para um conjunto de dados, a projeção do vetor $\vec{b}$ sobre o espaço coluna da matriz dos coeficientes $A$ corresponde a encontrar uma solução

\footnotetext{
${ }^{1}$ italonpaz@gmail.com

${ }^{2}$ fabianatsantana@gmail.com
} 
para $A \vec{x}=\vec{b}$ com erro mínimo. A abordagem utilizada foi alterar o problema de modo que não tenhamos que exigir que a equação matricial $A \vec{x}=\vec{b}$ seja satisfeita, em vez disso, procura-se um vetor solução $\vec{x}$ substituindo $\vec{b}$ por um vetor que esteja tão próximo de $A \vec{x}$ quanto possível. Tal vetor é a projeção ortogonal de $\vec{b}$ no espaço coluna de $A$, [1].

Pelo Teorema da Melhor Aproximação, se $W$ for o espaço coluna de $A$ com produto interno euclidiano de $R^{m}$, então a $\operatorname{proj}_{W} \vec{b} \in W$ satisfaz $\left\|\vec{b}-\operatorname{proj}_{W} \vec{b}\right\|<\|\vec{b}-\vec{w}\|$ para qualquer vetor $\vec{w} \in W$ distinto de $\operatorname{proj}_{W} \vec{b}$. Este teorema, cuja demonstração pode ser vista em [2], garante que a projeção de $\vec{b}$ em $W$ é a função que melhor se aproxima de $\vec{b} \notin W$ em $W$. Por outro lado, como $\vec{b}-A \vec{x}=\vec{b}-\operatorname{proj}_{W} \vec{b}$ é ortogonal à $W$ e $W$ é o espaço coluna de $A$, segue que $\vec{b}-A \vec{x}$ está no espaço nulo de $A^{T}$, pois o espaço nulo de $A^{T}$ e o espaço coluna são complementos ortogonais. Desse modo, uma solução de mínimos quadrados de $A \vec{x}=\vec{b}$ deve satisfazer $A^{T}(\vec{b}-A \vec{x})=\overrightarrow{0}$ ou $A^{T} A \vec{x}=A^{T} \vec{b}$, que é chamado de sistema normal associado à $A \vec{x}=\vec{b}$. Assim, o problema de encontrar uma solução de míminos quadrados foi reduzido a encontrar uma solução exata do sistema normal associado.

O estudo feito foi aplicado em obter uma estimativa da população brasileira no ano de 2020. Para isso, com base nos dados do Instituto Brasileiro de Geografia e Estatística, obtivemos o conjunto de dados $\left(x_{i}, y_{i}\right)$, onde os dados $x_{i}$ representam o ano de cada pesquisa realizada e os dados $y_{i}$ representa a quantidade de milhões de habitantes na respectiva data da pesquisa.

Ao aproximarmos o problema por polinômios de grau elevado, como o de grau 6, $p_{6}(x)=37762892-107152.51 x+112.44565 x^{2}-0.0507168 x^{3}+0.0000059 x^{4}+2.28210^{-9} x^{5}-$ $5.55210^{-13} x^{6}$, percebemos que os coeficientes de $x^{4}$ já começa a ter relevância pequena em comparação ao de $x^{3}$. No caso do polinômio de grau $4, p_{4}(x)=-49025107.0+100561.53 x-$ $77.330493 x^{2}+0.0264214 x^{3}-0.0000034 x^{4}$, o coeficiente de $x^{3}$ torna-se pequeno, mas não tanto quando comparado com os outros polinômios de graus maiores. Logo, a curva que apresenta uma aproximação satisfatoria, relevância dos coeficientes e comportamento gráfico satisfatório, aos dados apresentados é o polinômio de grau 4, tendo uma população estimada de 203.07 milhões de habitantes.

\section{Agradecimentos}

Este trabalho foi financiado pela PROPESQ-UFRN.

\section{Referências}

[1] H. Anton e R. C. Busby, Álgebra linear contemporânea, Porto Alegre: Bookman, (2006).

[2] H. Anton e C. Rorres, Álgebra linear com aplicações, Porto Alegre: Bookman, (2001).

[3] S. C. Chapra e R. P. Canale, Métodos numéricos para engenharia, São Paulo: McGraw-Hill, (2008). 